\title{
Y-Ba-Cu-O Nanostripes for Optical Photon Detection
}

\author{
G. P. Pepe,${ }^{1,2 *}$ L. Parlato, ${ }^{1,2}$ M. Ejrnaes ${ }^{2}$, R.Cristiano, ${ }^{2}$ R. Arpaia, ${ }^{3}$ F. Tafuri, ${ }^{2,4}$ D. Golubev, ${ }^{5}$ \\ T. Bauch, ${ }^{3}$ F. Lombardi, ${ }^{3}$ and Roman Sobolewski ${ }^{6,7}$ \\ ${ }^{1}$ Dipartimento di Fisica, Università degli Studi di Napoli Federico II, I-80125 Napoli, Italy \\ ${ }^{2}$ CNR-SPIN Institute of Superconductors, Innovative Materials and Devices, \\ UOS-Napoli, I-80125 Napoli, Italy \\ ${ }^{3}$ Quantum Device Physics Laboratory, Department of Microtechnology and Nanoscience, Chalmers \\ University of Technology, S-41296 Gothenburg, Sweden \\ ${ }^{4}$ Dipartimento di Ingegneria Industriale e dell'Informazione, Seconda Università di Napoli, \\ I-81031 Aversa (CE), Italy \\ ${ }^{5}$ Low Temperature Laboratory (OVLL), Aalto University School of Science, P.O. Box 13500, \\ SF-00076 Aalto, Finland \\ ${ }^{6}$ Institute of Electron Technology, PL-02668 Warszawa, Poland \\ ${ }^{7}$ Department of Electrical and Computer Engineering and Laboratory for Laser Energetics, \\ University of Rochester, NY 14627-0231, USA
}

\begin{abstract}
Nanowires of $\mathrm{Y}-\mathrm{Ba}-\mathrm{Cu}-\mathrm{O}$, with the thickness of $50 \mathrm{~nm}$ and the width ranging from $90 \mathrm{~nm}$ to $500 \mathrm{~nm}$ have been successfully grown on lanthanum aluminate substrates for photon detection experiments. The nanowires were up to 10$\mu \mathrm{m}$ long and formed a meander structure, covering the area of up to $30 \times 10 \mu \mathrm{m}^{2}$ with a fill factor of $50 \%$. The samples were excited using optical laser pulses at a $1550 \mathrm{~nm}$ wavelength and resulting photoresponse signals were measured as a function of both temperature and normalized bias current. Presence of two, distinct regimes in the photoresponse temperature dependence has been clearly evidenced, suggesting different physical mechanisms of the signal formation. Presented experimental results shed new light on prospects of implementation of high-temperature superconducting oxides in photon detection and counting.
\end{abstract}

Keywords: Superconducting nanostripes, high-temperature superconductors, optical photoresponse, superconducting single-photon detectors.

\section{INTRODUCTION}

Superconducting nanostripe single photon detectors (SNSPDs) are currently object of great interest in the field of quantum optics, quantum key distribution, and space telecommunication, because of their very high counting rates, extremely low dark counts, and high sensitivity for detection of single, infrared-to-visible photons. ${ }^{1-3}$ The best realizations are presently based on ultrathin nanostructures of low-temperature superconductors (LTSs), such as $\mathrm{NbN}$ or $\mathrm{NbTiN}$, operating in the temperature range of $1.7-5 \mathrm{~K}^{3-5}$ However, a significantly higher operating temperature is highly desired, especially for wide-spread commercial applications. High-temperature superconductors (HTSs), such as, e.g., $\mathrm{YBa}_{2} \mathrm{Cu}_{3} \mathrm{O}_{7}$-x (YBCO) exhibit not only high transition temperature $T_{\mathrm{C}}$, but also ultrafast (picosecond range) dynamics of nonequilibrium quasiparticles and high optical absorption, hence they seem to be good candidates for advanced SNSPDs. Unfortunately, several drawbacks in the present-day YBCO fabrication technology make the realization of YBCO-based SNSPDs a very challenging task.

Mechanism of superconductivity in HTS materials is quite different from the conventional electron-pairing scenario in metallic superconductors, so the resulting coherence length is ultrashort, making the photon-induced hotspot size negligibly small. Thus, HTS nanostructures suitable for photon-detection operation should be extremely small (even smaller than LTS SNSPDs). The latter is extremely challenging, since serious degradation of the superconducting properties typically occurs in YBCO structures when their thickness and/or width approach the nanometer scale, due to

*gpepe@na.infn.it

Photon Counting Applications 2015, edited by Ivan Prochazka, Roman Sobolewski, Ralph B. James, Proc. of SPIE Vol. 9504, 950406 · @ 2015 SPIE · CCC code: 0277-786X/15/\$18 · doi: 10.1117/12.2179677 
the damage of the nanostructure surface and edges, as well as the oxygen out-diffusion. ${ }^{6-9}$ Another important issue is that a typical current-voltage (I-V) characteristics of an YBCO nanostripe exhibits a gradual, rather than sharp transition from the superconducting to the resistive state, due to the presence of a, so-called, flux-flow region, where a resistive state appears, but the superconductivity is still present in the sample. ${ }^{10}$ This last feature, together with the ultrashort recovery time of the superconducting state in HTS materials, results that the mechanism of the YBCO photoresponse is quite different than that in the LTS case. ${ }^{11}$ In HTS materials, nanostripes are extremely two-dimensional (2D) superconductors with respect to both the coherence length and the London penetration depth, hence, microscopic mechanisms, such as quantum-mechanical or thermally-activated phase slip centers, unbinding of vortex-antivortex pairs (VAPs), quantum mechanical tunneling of vortices, or thermal excitation of single vortices across the stripe edge barrier and their subsequent dissipative movement across the stripe width, are expected to play a very significant role in the photoresponse signal resulting from absorption of a photon.

In this work, we present the experimental results concerning the photoresponse of very thin YBCO nanostripes, exposed to optical laser pulses at $1550 \mathrm{~nm}$ wavelength and operated at temperatures down to $T=4 \mathrm{~K}$. The obtained results open the prospect of realizing photon detectors based on YBCO stripes, especially the ones with even lower thicknesses that could exhibit hysteretic I-V curves, as predicted by the theoretical models.

\section{EXPERIMENTAL DETAILS}

\section{Sample fabrication}

We have fabricated a series of YBCO nanostripes with widths varying from 90 to $500 \mathrm{~nm}$ and lengths of up to $10 \mu \mathrm{m}$. As is shown in Fig. 1(a), our test structures were segmented meanders, covering areas up to $30 \times 10 \mu \mathrm{m},{ }^{2}$ with the distance between the stripes fixed to $500 \mathrm{~nm}$, what corresponded to a fill factor of $50 \%$. To minimize current crowding effects, which could be a source of reduction of the critical current $I_{\mathrm{C}}$ in our nanostructures, the edges between the stripes were wider and had rounded shapes [see Fig. 1(b)]. Our meander structures we patterned in 50-nm-thick, YBCO films deposited using a pulsed laser deposition method in $\mathrm{O}_{2}$ pressure of $0.6 \mathrm{mbar}$ and at temperature of $800{ }^{\circ} \mathrm{C}$ on lanthanum aluminate substrates. The films exhibited excellent structural and transport properties with about $0.3^{\circ}$ full-width-at-halfmaximum rocking curves of the (005) reflection and surface roughness of only $2 \mathrm{~nm}$. The value of $T_{\mathrm{C}}$ was $89 \mathrm{~K}$ for all samples. Patterning of nanostructures was done using same procedure as reported in ${ }^{12,13}$ with a 50 -nm-thick Au layer deposited by e-beam evaporation on top of the YBCO layer. The Au film acts as a cap, protecting the structure against damages occurring during the processing steps, and was completely removed by a $\mathrm{Ar}^{+}$etching at the end of the fabrication procedure. In particular, our meander structures had a series/parallel stripe configuration, consisting of a serial connection of blocks (7, 9, 11 and 13 blocks) each formed by 3 nanostripes in parallel [see Fig. 1(a)]. As a consequence of this geometry, we expect photoresponse signal to have an enhanced amplitude, since it is proportional to the current corresponding to the sum of currents in all our parallel nanostripes. ${ }^{14}$ Moreover, parallel connection of nanostripes reduces the total kinetic inductance of the structure, resulting in the faster photoresponse signal.

\section{Experimental results}

Transport properties of nanostripes have been characterized in terms of I-V characteristics. We measured the critical current density $J_{\mathrm{C}}$ of up to $\sim 10^{6} \mathrm{~A} / \mathrm{cm}^{2}$ at $5 \mathrm{~K}$. In this temperature range, the $\mathrm{I}-\mathrm{V}$ curve of an ultrathin $\mathrm{YBCO}$ nanostructure resembled the one routinely observed in LTS-based nanostripes with a hysteresis loop close to $I_{\mathrm{C}}$.
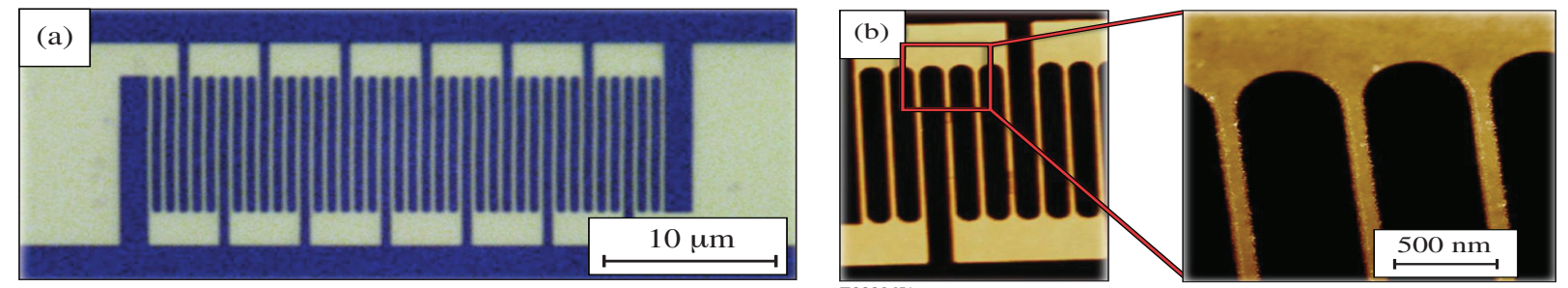

Figure 1. (a) Micrograph of a device formed by serial/parallel connections of 300-nm-wide YBCO nanostripes; (b) An AFM scan of several 130-nm-wide YBCO nanowires. The inset shows in detail rounded corners between the stripes, designed to avoid reduction of $I_{\mathrm{C}}$ caused by the current crowding effect. 
Photoresponse experiments have been carried out by mounting the devices in a liquid helium, continuous-flow cryostat with an optical access. The test structures were illuminated by optical laser pulses with a $1550 \mathrm{~nm}$ wavelength. ${ }^{15}$ The pulses had both rise and fall times of $\sim 3 \mathrm{~ns}$, duration of $500 \mathrm{~ns}$, and a nominal intensity of about $50 \mathrm{nW} / \mu \mathrm{m}^{2}$. When the meanders were biased far below $I_{\mathrm{C}}$, no measureable photoresponse was detected. A voltage signal appeared only when the device was either biased slightly below $I_{\mathrm{C}}$ or in the mixed state. The maximum of the voltage response, however, corresponded to the bias $I_{\mathrm{b}} \approx I_{\mathrm{C}}$ and afterward the signal amplitude decreased with the $I_{\mathrm{b}}$ increase, until it either vanished completely or reached a saturation value (few $\mu \mathrm{V})$ at $I_{\mathrm{b}}>I_{\mathrm{C}}$.

Voltage pulses unambiguously corresponding to the incident laser pulses have been detected from $4.9 \mathrm{~K}$ up to $T_{\mathrm{C}}$, and an example of such a photoresponse is shown in Fig. 2(a), where the measured signal (red dots) is overlaid with the inverse of laser pulses used to illuminate the device. We note that the detected transients roughly overlap with the optical pulses, however, their rise and fall times are significantly longer and equal to $\sim 50 \mathrm{~nm}$.

Finally, Fig. 2(b) shows the amplitude of the photoresponse signal as a function of the normalized bias current at two different temperatures for a $500-\mathrm{nm}$ nanostripe structure. We observe that at low temperatures ( $T=4.9 \mathrm{~K}$; black squares) the signal has a sharp peak very close to $I_{\mathrm{b}}=I_{\mathrm{C}}$ and quickly decreases at higher bias levels. On the other hand at high temperatures $\left(T=70 \mathrm{~K}\right.$; red circles), the behavior is very different: at $I_{\mathrm{b}} \approx I_{\mathrm{C}}$, the photoresponse amplitude is negligibly small and then, gradually, increases, reaching a saturated maximum value at $I_{\mathrm{b}} \gg I_{\mathrm{C}}$. The presence of two different regimes indicates that the YBCO nanostripe photoresponse mechanism at the superconducting state very close to $I_{\mathrm{C}}$ is very different from the one at the mixed resistive state. We also remind here that at $I_{\mathrm{b}}<<I_{\mathrm{C}}$, we did not observe any photoresponse signal.
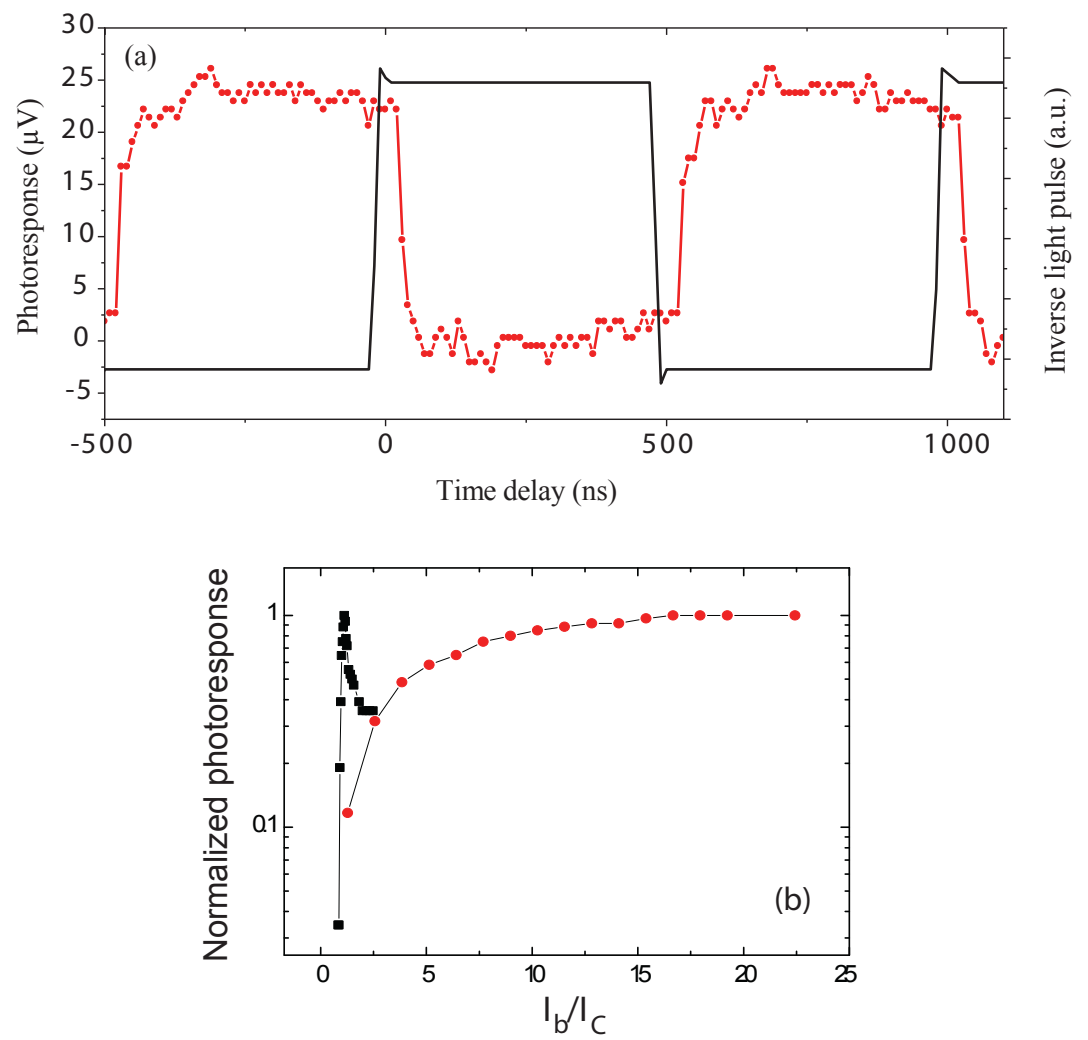

Figure 2. (a) A photoresponse signal (red points) measured at $T=4.9 \mathrm{~K}$ with $I_{\mathrm{b}}$ slightly below $I_{\mathrm{C}}\left(I_{b} / I_{c}=0.99\right)$ overlaid on top of the inverse of optical pulses; (b) Normalized photoresponse amplitudes vs the normalized bias current, collected at $T=4.9 \mathrm{~K}$ (black squares) and at $T=70 \mathrm{~K}$ (red circles) for the same tested YBCO nanostructure. 


\section{DISCUSSION AND CONCLUSIONS}

The observed photoresponse behavior in YBCO nanostructures is markedly different from that observed in LTS-based, SNSPDs. In metallic nanostripes, I-V curves are highly hysteretic and a large voltage swing occurs just above $I_{\mathrm{C}}$, bringing the structure into the fully normal state. In the SNSPD operation, photon absorption creates in a nanostripe a hotspot resulting in resistive state with the normal resistance much larger than the external load impedance $R_{\mathrm{L}}$ of the read-out circuit. As a result, the whole $I_{\mathrm{b}}$ is redirected into the readout branch of the electronic circuit and the voltage output signal is proportional to the $I_{\mathrm{b}} \times R_{\mathrm{L}}$ product. At the same time, for biases $I_{\mathrm{b}}>I_{\mathrm{C}}$ the device remains in the fully resistive state and photon absorption makes no impact on its resistance.

In our YBCO-based nanostripes, the resistive branch of the experimental I-V characteristics represents a mixed or intermediate state, where both the superconducting and normal domains coexist. Presently, YBCO nanostructures are relatively large of as compared to their coherence length, so a hotspot created after the photon absorption is way too small to drive a whole nanostripe into the normal state, and can only generate an intermediate state, where most likely the fluxon dynamics starts to play a fundamental role. The latter is corroborated by a theoretical study based on the heat transport in YBCO nanostripes that predicts that formation of normal hotspots should occur at significantly thinner (below $10 \mathrm{~nm}$ ) nanostructures than the ones studied in our experiments. Analyzing our experimental data, we can speculate that a non-bolometric component of the photoresponse is likely to be present at low temperatures (well below $\sim 50 \mathrm{~K}$ ), where high-amplitude signals with relatively fast rise times were recorded. At high temperatures (above $\sim 50 \mathrm{~K}$ ) we cannot rule out a heating effect as a dominant component of the YBCO photoresponse signal. The behavior is, actually, similar to that observed in Josephson junctions in the presence of thermal noise. ${ }^{16}$

\section{ACKNOWLEDGEMENT}

The authors acknowledge the European Union COST Action MP1201.

\section{REFERENCES}

[1] G. N. Gol'tsman, O. Okunev, G. Chulkova, A. Lipatov, A. Semenov, K. Smirnov, B. Voronov, A. Dzardanov, C. Williams, and R. Sobolewski, Appl Phys Lett 79 (2001) 705

[2] C. M. Natarajan, M. G. Tanner, and R. H. Hadfield, Sc. \& Tech. 25, 063001 (2012) and references therein.

[3] R. H. Hadfield, Nature Photonics 3696 (2009) and references therein.

[4] F. Marsili, V. B Verma, J. A. Stern, S. Harrington, A. E. Lita, T. Gerrits, I. Vayshenker, and B. Baek, Nat. Photonics 7, 210 (2013).

[5] Yu P Korneeva et al. , Supercond. Sci. Technol., 27095012 (2014)

[6] P. Larsson, B. Nilsson, and Z. G. Ivanov, J. Vac. Sci. Technol. B 18 (2000) 25

[7] S. Nawaz, T. Bauch, and F. Lombardi, IEEE Trans. Appl Supercond. 21 (2011) 164

[8] F. Tafuri et al, J. Supercond. Nov. Magn. 26 (2013) 21

[9] D. Gustafsson, et al., Nature Nanotechnol 8 (2013) 25

[10] D. Golubev, F. Lombardi, T. Bauch, Physica C (2014)

[11] D. Pan, G. P. Pepe, V. Pagliarulo, C. De Lisio, L. Parlato, M. Khafizov, I. Komissarov, and R. Sobolewski, Phys. Rev. B 78 (2008) 174503

[12] S. Nawaz, R. Arpaia, T. Bauch, and F. Lombardi, Physica C 495 (2013) 33

[13] R. Arpaia, S. Nawaz, F. Lombardi, and T. Bauch, IEEE Trans. Appl. Supercond. 23 (2013) 1101505

[14] M. Ejrnaes, A. Casaburi, O. Quaranta, S. Marchetti, A. Gaggero, F. Mattioli, R. Leoni, S. Pagano and R. Cristiano, Supercond. Sci. Technol, 22, 055006 (2009)

[15] R. Arpaia, M. Ejrnaes, L. Parlato, F. Tafuri, R. Cristiano, D. Golubev, R. Sobolewski, T. Bauch, F. Lombardi, G. P. Pepe, Physica C 509 (2015) 16

[16] V. Ambegaokar and B. I. Halperin, Phys. Rev. Lett. 22 (1969) 1364 